\title{
An Updated Nomogram for Predicting Invasiveness in Preoperative Ductal Carcinoma In Situ of the Breast
}

\author{
Sanghwa Kim ${ }^{1 *}$, Jihong Kim ${ }^{2 *}$, Hyung Seok Park², Ha Yan Kim³ ${ }^{3}$ Kwanbum Lee², Jeea Lee ${ }^{2}$, \\ Haemin $\mathrm{Lee}^{2}$, Jee Ye Kim², Seung Il Kim², Young Up $\mathrm{Cho}^{2}$, and Byeong-Woo Park ${ }^{2}$ \\ ${ }^{1}$ Department of Breast and Endocrine Surgery, Hallym University Sacred Heart Hospital, Hallym University College of Medicine, Anyang; \\ ${ }^{2}$ Department of Surgery, Yonsei University College of Medicine, Seoul; \\ ${ }^{3}$ Biostatistics Collaboration Unit, Department of Biomedical Systems Informatics, Yonsei University College of Medicine, Seoul, Korea.
}

Purpose: To validate and update a nomogram for predicting ductal carcinoma in situ (DCIS) upstaging in preoperative biopsy. Materials and Methods: Medical records of 444 preoperative DCIS patients were evaluated and used to validate a previous version of the Severance nomogram for predicting DCIS upstaging in preoperative biopsy. Patients were divided into two groups according to the final postoperative pathology. Univariate and multivariate analyses with the chi-square test, Student's t-test, and binary logistic regression method identified new significant variables. The updated nomogram was evaluated with the C-index and Hosmer-Lemeshow goodness of fit test.

Results: The area under a receiver operating characteristic curve for comparison with the previous nomogram was 0.48 . In postoperative pathology, the pure DCIS and invasive cancer groups comprised 345 and 99 cases, respectively. Approximately $22.3 \%$ of patients preoperatively diagnosed with DCIS were upstaged to invasive cancer. Significant variables in the univariate analysis were operation type, human epidermal growth factor receptor 2 overexpression, comedo necrosis, sonographic mass, mammographic mass, preoperative biopsy method, and suspicious microinvasion in preoperative biopsy. In multivariate analysis, operation type, sonographic mass, mammographic mass, and suspicious microinvasion were risk factors for upstaging. The updated model with these variables showed moderate discrimination and was appropriate in the calibration test.

Conclusion: The previous nomogram did not effectively discriminate upstaging of preoperative DCIS in an independent cohort. An updated version of the nomogram appears to provide more accurate information for predicting preoperative DCIS upstaging.

Key Words: Breast neoplasms, prediction, ductal carcinoma in situ, upstaging

\section{INTRODUCTION}

Invasive breast cancer can be transferred to the lymph nodes, such that evaluation of axillary lymph node metastasis using

Received: May 17, 2019 Revised: August 26, 2019

Accepted: September 6, 2019

Corresponding author: Hyung Seok Park, MD, PhD, Department of Surgery, Yonsei University College of Medicine, 50-1 Yonsei-ro, Seodaemun-gu, Seoul 03722, Korea.

Tel: 82-2-2228-2100, Fax: 82-2-313-8289, E-mail: imgenius@yuhs.ac

*Sanghwa Kim and Jihong Kim contributed equally to this work.

-The authors have no potential conflicts of interest to disclose.

(C) Copyright: Yonsei University College of Medicine 2019

This is an Open Access article distributed under the terms of the Creative Commons Attribution Non-Commercial License (https://creativecommons.org/licenses/ by-nc/4.0) which permits unrestricted non-commercial use, distribution, and reproduction in any medium, provided the original work is properly cited. clinical examination, sentinel lymph node biopsy (SLNB), or axillary lymph node dissection (ALND) is necessary: ${ }^{1}$ SLNB can be used to identify axillary lymph node metastasis in patients with early breast cancer. ${ }^{2,3}$ Ductal carcinoma in situ (DCIS) is a localized tumor within the ducts without invasion of the basement membrane. By definition, pure DCIS has no potential to spread tumor cells to the axillary lymph nodes. Therefore, according to international guidelines, routine use of SLNB and ALND is not recommended for patients with DCIS. ${ }^{1,3}$ However, in actual practice, controversy remains as to whether SLNB should be performed in DCIS diagnosed via preoperative biopsy. ${ }^{4-6}$ A major concern for surgeons is that additional axillary surgery for the evaluation of metastasis to the axillary lymph nodes may be necessary in cases with preoperative DCIS when invasive foci are identified in the permanent pathology. When DCIS is preoperatively diagnosed with a core needle biopsy (CNB), 
an invasive component can be under-estimated because of sampling limitations. In a meta-analysis of 52 studies, approximately $26 \%$ of cases of DCIS diagnosed using CNB were upstaged to invasive cancer. ${ }^{7}$ Thus, a previous study suggested routine use of SLNB in patients with DCIS. ${ }^{8}$ However, SLNB is not a riskfree procedure, and although it has lower morbidity rates than ALND, a risk of complications remains (e.g., seroma, lymphedema of the arm, pain, and sensory deterioration in the axillary region). Therefore, shared decision-making in terms of performing or omitting SLNB at the time of surgery for patients with preoperative DCIS is crucial.

To enhance shared decision-making, predicting upstaging of preoperative DCIS can be helpful for patients and surgeons. Several studies have been conducted to establish a nomogram for predicting the possibility of preoperative DCIS upstaging to invasive ductal carcinoma after surgery. ${ }^{9-14}$ However, previous nomograms have had several limitations, including the lack of external validation, lack of clinical utility based on sophisticated variables, and lack of popularity or accessibility. Therefore, we conducted this study to validate a previous nomogram using an independent external cohort, to reanalyze factors associated with DCIS underestimation, and to establish an updated nomogram.

\section{MATERIALS AND METHODS}

\section{Patient cohort}

We used the Breast Cancer Registry database of Severance Hospital, Yonsei University Health System, to perform a retrospective analysis. The computerized medical database was constructed in MS Access (Microsoft, Seattle, WA, USA) and contained information on patient clinical characteristics, pathologic data of preoperative or postoperative evaluations, treatment methods, recurrence data, preoperative evaluation findings, including those of physical examination, mammography, and ultrasonography, and follow-up data.

From 2009 to 2012, 538 patients were preoperatively diagnosed with DCIS and underwent definitive surgery. Patients were ex-

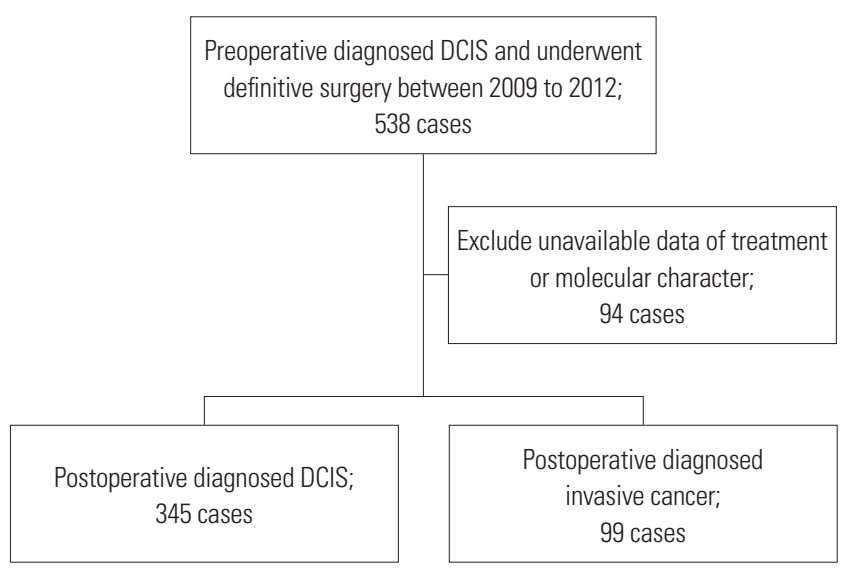

Fig. 1. Patient cohort. DCIS, ductal carcinoma in situ. cluded from the analysis if they had no available data on the type of treatment or molecular characteristics, such as hormone receptors and human epidermal growth factor receptor 2 (HER2) status (Fig. 1).

Patients underwent breast-conserving surgery or mastectomy at their surgeons' discretion based on the size, location, and multiplicity of the tumor, as well as according to the patient's preference. Along with breast surgery, axillary lymph node evaluation was performed via SLNB and/or standard level I/II ALND. After surgery, patients who underwent breast-conserving surgery received adjuvant radiotherapy with a median boost dose of $10 \mathrm{~Gy}$ that covered the whole breast with or without the regional nodal area. Adjuvant endocrine therapy was administered, if indicated.

Patient characteristics, including age, preoperative biopsy methods, clinical findings, pathologic findings, and treatment methods, were reviewed. We enrolled 115 additional patients who underwent surgery from 2013 to 2015 for independent external validation of the nomogram. This study was reviewed and approved by the Institutional Review Board of Severance Hospital, Yonsei University Health System (IRB No. 4-2018-1027).

\section{Preoperative biopsy methods}

Patients were categorized according to preoperative biopsy into $\mathrm{CNB}$ and vacuum-assisted biopsy (VAB) groups. CNB was performed with a 14-gauge semi-automated core needle under ultrasound guidance. In general, four to six core pieces were obtained by radiologists specializing in breast imaging during CNB. VAB was performed with an 8- or 11-gauge vacuum-assisted large CNB system under stereotactic or ultrasound guidance.

\section{Clinical examination and radiology}

Preoperative imaging evaluation including mammography and ultrasonography was performed by experienced radiologists, and the initial report of the preoperative imaging studies was analyzed in conjunction with the final pathology to review the correlation between imaging studies and the final pathology. The final assessment of the imaging studies was recorded with the Breast Image and Reporting Data System developed by the American College of Radiology (https://www.acr.org/-/media/ACR/ Files/RADS/BI-RADS/BIRADS-Reference-Card.pdf). The size of lesions on ultrasonography was reviewed using medical records. A preoperative physical examination was performed by experienced surgeons, and a palpable mass was described with or without information on the location or size of the lesions in the medical database.

\section{Histopathology}

Final pathology records were reviewed to analyze histopathological variables, including suspicious invasive foci in preoperative pathologic findings, presence of comedo necrosis, DCIS nuclear grade, Van Nuys Prognostic Index, hormone receptor status, amplification of HER2/neu, Ki-67 proliferative index lev- 
el, and number of metastatic axillary lymph nodes. Comedo necrosis was considered to be either absent or present. Nuclear grade was determined according to the College of American Pathologists guidelines. Estrogen receptor (ER), progesterone receptor (PR), and HER2/neu expression in primary breast cancer was evaluated based on formalin-fixed, paraffin-embedded whole sections of surgically resected breast cancer specimens using immunohistochemistry (IHC). The cut-off value for ER and PR positivity was over $1 \%$ staining in IHC. A staining level of $3+$ on IHC was defined as positive for HER2/neu overexpression and/or when amplification was evident in a fluorescence in situ hybridization assay.

\section{Statistical analysis}

Using data from 444 patients with preoperative DCIS, we analyzed categorical variables using the chi-square test or Fisher's exact test. Continuous variables were analyzed using Student's t-test. The significant factors estimated by the aforementioned analyses were confirmed using univariate logistic regression. To identify factors independently associated with invasion, binary logistic regression analysis adjusted for significant factors in the univariate analysis was performed. To reduce the risk of multicollinearity, some of the closely correlated variables were excluded from the multivariate analysis. The significant factors from the multivariate analysis were used to construct the updated nomogram.

Nomogram performance was quantified with respect to discrimination and calibration using the C-index and HosmerLemeshow goodness of fit test in internal validation. The nomogram was externally validated using independent data sets.
Discrimination was quantified using the means of the area under the receiver operating characteristic (ROC) curve. The calibration of the model was assessed graphically, and the area under the curve (AUC) was estimated. $p$ values $<0.05$ were considered significant; all tests were two-sided. Statistical analyses were conducted using commercially available statistical software (SPSS Statistics 24; IBM Corp., Armonk, NY, USA).

\section{RESULTS}

\section{Clinicopathologic characteristics}

Patient and tumor characteristics are shown in Table 1. A total of 345 patients were found to have pure DCIS, whereas 99 patients had invasive cancer. Approximately one-fifth (22.3\%) of preoperative DCIS cases was upstaged to invasive cancer in the final pathology. Axillary evaluation was performed in almost all patients (99.1\%). Axillary lymph node metastasis was found in $2.0 \%$ of patients in the DCIS group and in $13.1 \%$ of patients in the invasive cancer group. Age at diagnosis ( $p=0.887)$, proportion of palpability of the lesion in physical examination ( $p=0.501)$, presence of calcification on imaging (sono calcification $p=0.659$, mammo calcification $p=0.126$ ), and ER status ( $p=0.151$ ) did not differ significantly between the groups.

Factors associated with prediction of invasive cancer Table 1 shows the predictive factors associated with invasiveness in 444 patients with preoperative DCIS. Operation type $(p<0.001)$, presence of comedo necrosis $(p<0.001)$, existence of mass finding on sonography $(p<0.001)$ or mammography $(p=0.001)$, pre-

Table 1. Clinicopathologic Characteristics of the Training Data Set ( $n=444)$

\begin{tabular}{|c|c|c|c|c|c|}
\hline & \multicolumn{2}{|c|}{ DCIS ( $n=345)$} & \multicolumn{2}{|c|}{ Invasive cancer (n=99) } & \multirow{2}{*}{$p$ value } \\
\hline & $\mathbf{n}$ & $\%$ & $\mathbf{n}$ & $\%$ & \\
\hline Age at diagnosis & & & & & 0.887 \\
\hline Same or less than 50 & 184 & 53.3 & 52 & 52.5 & \\
\hline Older than 50 & 161 & 46.7 & 47 & 47.5 & \\
\hline BCS probability & & & & & $<0.001$ \\
\hline Yes (BCS) & 177 & 51.3 & 30 & 30.3 & \\
\hline No (Mastectomy) & 168 & 48.7 & 69 & 69.7 & \\
\hline Estrogen receptor & & & & & 0.151 \\
\hline Negative & 106 & 30.7 & 38 & 38.4 & \\
\hline Positive & 239 & 69.3 & 61 & 61.6 & \\
\hline Progesterone receptor & & & & & 0.503 \\
\hline Negative & 182 & 52.8 & 56 & 56.6 & \\
\hline Positive & 163 & 47.2 & 43 & 43.4 & \\
\hline HER2/neu & & & & & 0.028 \\
\hline Negative & 112 & 32.5 & 44 & 44.4 & \\
\hline Overexpression & 233 & 67.5 & 55 & 55.6 & \\
\hline KI67 & & & & & 0.104 \\
\hline Same or less than 14 & 336 & 97.4 & 99 & 100.0 & \\
\hline More than 14 & 9 & 2.6 & 0 & 0.0 & \\
\hline
\end{tabular}


Sanghwa Kim, et al.

Table 1. Clinicopathologic Characteristics of the Training Data Set ( $n=444)$ (Continued)

\begin{tabular}{|c|c|c|c|c|c|}
\hline & & & Inva & $n=99$ ) & n ulu \\
\hline & $\mathbf{n}$ & $\%$ & $\mathbf{n}$ & $\%$ & $p$ value \\
\hline Grade & & & & & 0.838 \\
\hline Low/intermediate & 213 & 61.7 & 60 & 60.6 & \\
\hline High & 132 & 38.3 & 39 & 39.4 & \\
\hline Van Nuys group & & & & & 0.092 \\
\hline Group 1 & 9 & 29.0 & 2 & 22.2 & \\
\hline Group 2 & 6 & 19.4 & 5 & 55.6 & \\
\hline Group 3 & 16 & 51.6 & 2 & 22.2 & \\
\hline Comedo necrosis & & & & & $<0.001$ \\
\hline Non-comedo & 91 & 26.4 & 46 & 46.5 & \\
\hline Comedo & 254 & 73.6 & 53 & 53.5 & \\
\hline USG mass & & & & & $<0.001$ \\
\hline Absent & 108 & 31.3 & 13 & 13.1 & \\
\hline Present & 237 & 68.7 & 86 & 86.9 & \\
\hline USG calcification & & & & & 0.659 \\
\hline Absent & 183 & 53.0 & 55 & 55.6 & \\
\hline Present & 162 & 47.0 & 44 & 44.4 & \\
\hline USG category & & & & & 0.092 \\
\hline Category 1-3 & 11 & 3.2 & 1 & 1.0 & \\
\hline Category 4-5 & 328 & 95.1 & 93 & 93.9 & \\
\hline Category 6 & 6 & 1.7 & 5 & 5.1 & \\
\hline MMG mass & & & & & 0.001 \\
\hline Absent & 294 & 85.2 & 70 & 70.7 & \\
\hline Present & 51 & 14.8 & 29 & 29.3 & \\
\hline MMG calcification & & & & & 0.126 \\
\hline Absent & 85 & 24.6 & 32 & 32.3 & \\
\hline Present & 260 & 75.4 & 67 & 67.7 & \\
\hline MMG category & & & & & 0.339 \\
\hline Category 1-3 & 21 & 6.3 & 3 & 3.1 & \\
\hline Category 4-5 & 298 & 90.0 & 92 & 94.8 & \\
\hline Category 6 & 12 & 3.6 & 2 & 2.1 & \\
\hline Biopsy method & & & & & $<0.001$ \\
\hline FNAB/core needle biopsy & 220 & 63.8 & 83 & 83.8 & \\
\hline VAB/stereotactic biopsy & 125 & 36.2 & 16 & 16.2 & \\
\hline Suspicious invasion in preoperative biopsy & & & & & $<0.001$ \\
\hline Absent & 333 & 96.5 & 85 & 85.9 & \\
\hline Present & 12 & 3.5 & 14 & 14.1 & \\
\hline Palpability & & & & & 0.501 \\
\hline Non-palpable & 86 & 24.9 & 28 & 28.3 & \\
\hline Palpable & 259 & 75.1 & 71 & 71.7 & \\
\hline Axillary evaluation & & & & & $<0.001$ \\
\hline Not done & 3 & 0.9 & 1 & 1.0 & \\
\hline SLNB & 78 & 22.6 & 17 & 17.2 & \\
\hline ALND & 264 & 76.5 & 81 & 81.8 & \\
\hline Axillary lymph node metastasis & & & & & $<0.001$ \\
\hline Absent & 338 & 98.0 & 86 & 86.9 & \\
\hline Present & 7 & 2.0 & 13 & 13.1 & \\
\hline
\end{tabular}

DCIS, ductal carcinoma in situ; BCS, breast-conserving surgery; HER2/neu, human epidermal growth factor receptor 2; USG, breast sonography; MMG, mammography; FNAB, fine needle aspiration biopsy; VAB, vacuum-assisted biopsy; SLNB, sentinel lymph node biopsy; ALND, axillary lymph node dissection. 
operative biopsy method $(p<0.001)$, and suspicious microinvasion in preoperative biopsy $(p<0.001)$ were significantly related to upstaging to invasive cancer in the postoperative pathology.

Univariate logistic regression analysis of the 444 patients without missing data revealed seven significant variables: operation type [odds ratio $(\mathrm{OR})=2.42,95 \%$ confidence interval $(\mathrm{CI})=1.50^{-}$ $3.91, p<0.001]$, HER2 overexpression ( $\mathrm{OR}=0.6,95 \% \mathrm{CI}=0.38-0.95$, $p=0.028$ ), presence of comedo necrosis in the tumor ( $\mathrm{OR}=0.41$, $95 \% \mathrm{CI}=0.26-0.66, p<0.001$ ), sonographic mass ( $\mathrm{OR}=3.01$, $95 \%$ $\mathrm{CI}=1.61-5.64, p<0.001)$, mammographic mass ( $\mathrm{OR}=2.39,95 \%$ $\mathrm{CI}=1.41-4.04, p=0.001), \mathrm{VAB} /$ stereotactic biopsy as the preoperative biopsy method ( $\mathrm{OR}=0.34,95 \% \mathrm{CI}=0.19-0.61, p<0.001$ ), and presence of suspicious microinvasive foci in preoperative biopsy ( $\mathrm{OR}=4.58,95 \% \mathrm{CI}=2.04-10.24, p<0.001$ ) (Table 2).

In multivariate analysis, operation type $(\mathrm{OR}=3.46,95 \% \mathrm{CI}=2.02-$ 5.91, $p<0.001$ ), sonographic mass ( $\mathrm{OR}=2.75,95 \% \mathrm{CI}=1.40-5.38$, $p=0.003$ ), mammographic mass ( $\mathrm{OR}=2.23,95 \% \mathrm{CI}=1.24-4.01$, $p=0.007$ ), and suspicious microinvasion in preoperative biopsy (OR=3.96, 95\% CI=1.59-9.84, $p=0.003)$ were risk factors for upstaging to invasive cancer after surgery. In cases of DCIS with HER2 overexpression ( $\mathrm{OR}=0.55,95 \% \mathrm{CI}=0.33-0.93, p=0.024)$ and comedo necrosis ( $\mathrm{OR}=0.4,95 \% \mathrm{CI}=0.24-0.67, p<0.001)$, a diagnosis of pure DCIS after surgery was highly likely (Table 3 ).

\section{Nomogram establishment and validation}

The updated nomogram is illustrated in Fig. 2. The detailed establishment process of the nomogram was described in a previous study. ${ }^{10}$ The valuation of the updated model with the six significant variables showed moderate discrimination (C-in$\mathrm{dex}=0.75)$ and was appropriate in a calibration test $(p=0.452)$. External validation with an independent data set of 115 patients showed moderate to strong discrimination, with a C-index of 0.66 , and good fit, with a calibration test result of 0.92 . The ROC curve is illustrated in Fig. 3, and the AUC was 0.621 (95\% CI= 0.496-0.746).

\section{DISCUSSION}

It is important to predict the probability of invasive cancer before surgery, since it has a large impact on the range of surgery and the complications that patients should endure. This process can facilitate shared decision making between surgeons and patients. Therefore, more accurate and accessible methods are needed to predict invasiveness in preoperative diagnosed DCIS. In 2013, we published a nomogram that predicted the invasiveness of breast cancer diagnosed as pre-operative DCIS, and several other studies utilized it in the past few years. ${ }^{10,12-14}$ However, since the previous model had some limitations, it had to be updated using recent data. Therefore, we conducted this study to update the new nomogram. This updated nomogram was found to be more accurate in predicting invasive potential than the previous model and to be potentially more helpful for many surgeons and patients.

A previous study established a nomogram to predict the likelihood of postoperative invasive ductal carcinoma in DCIS at

Table 2. Univariate Logistic Regression Analysis of the Training Data Set

\begin{tabular}{|c|c|c|c|}
\hline & OR & $95 \% \mathrm{Cl}$ & $p$ value \\
\hline Age at diagnosis & & & 0.887 \\
\hline Same or less than 50 & Ref. & & \\
\hline Older than 50 & 1.03 & $0.66-1.62$ & \\
\hline BCS probability & & & $<0.001$ \\
\hline Yes (BCS) & Ref. & & \\
\hline No (Mastectomy) & 2.42 & $1.50-3.91$ & \\
\hline Estrogen receptor & & & 0.152 \\
\hline Negative & Ref. & & \\
\hline Positive & 0.71 & $0.45-1.13$ & \\
\hline Progesterone receptor & & & 0.502 \\
\hline Negative & Ref. & & \\
\hline Positive & 0.86 & $0.55-1.35$ & \\
\hline HER2/neu & & & 0.028 \\
\hline Negative & Ref. & & \\
\hline Overexpression & 0.6 & $0.38-0.95$ & \\
\hline Grade & & & 0.837 \\
\hline Low/intermediate & Ref. & & \\
\hline High & 1.05 & $0.66-1.66$ & \\
\hline Comedo necrosis & & & $<0.001$ \\
\hline Non-comedo & Ref. & & \\
\hline Comedo & 0.41 & $0.26-0.66$ & \\
\hline USG mass & & & 0.001 \\
\hline Absent & Ref. & & \\
\hline Present & 3.01 & $1.61-5.64$ & \\
\hline USG calcification & & & 0.658 \\
\hline Absent & Ref. & & \\
\hline Present & 0.9 & $0.58-1.42$ & \\
\hline MMG mass & & & 0.001 \\
\hline Absent & Ref. & & \\
\hline Present & 2.39 & $1.41-4.04$ & \\
\hline MMG calcification & & & 0.126 \\
\hline Absent & Ref. & & \\
\hline Present & 0.68 & $0.42-1.11$ & \\
\hline Biopsy method & & & $<0.001$ \\
\hline FNAB/core needle biopsy & Ref. & & \\
\hline VAB/stereotactic biopsy & 0.34 & $0.19-0.61$ & \\
\hline Suspicious invasion in preoperative biopsy & & & $<0.001$ \\
\hline Absent & Ref. & & \\
\hline Present & 4.58 & $2.04-10.24$ & \\
\hline Palpability & & & 0.500 \\
\hline Non-palpable & Ref. & & \\
\hline Palpable & 0.84 & $0.51-1.39$ & \\
\hline
\end{tabular}

$\mathrm{OR}$, odds ratio; $\mathrm{Cl}$, confidence interval; BCS, breast-conserving surgery; HER2/neu, human epidermal growth factor receptor 2; USG, breast sonography; MMG, mammography; FNAB, fine needle aspiration biopsy; VAB, vacuum-assisted biopsy. 
diagnosis. ${ }^{10,15}$ The application of the previous nomogram was checked by Jakub, et al. ${ }^{13}$ in their study, which showed poor calibration ( $p=0.002)$. We also performed an external validation of the previous nomogram using independent datasets, and it did not show high performance. The AUC of the ROC curve for comparison with the previous version of the nomogram was 0.48

Table 3. Multivariate Logistic Regression Analysis of Factors associated with Upstaging of Preoperative Ductal Carcinoma In Situ in the Training Data Set

\begin{tabular}{lccc}
\hline & OR & 95\% CI & p value \\
\hline $\begin{array}{l}\text { BCS probability } \\
\text { Yes (BCS) }\end{array}$ & Ref. & & $<0.001$ \\
No (Mastectomy) & 3.46 & $2.02-5.91$ & \\
HER2/neu & & & 0.024 \\
$\quad$ Negative & Ref. & \\
$\quad$ Overexpression & 0.55 & $0.33-0.93$ & \\
Comedo necrosis & & & $<0.001$ \\
$\quad$ Non-comedo & Ref. & \\
$\quad$ Comedo & 0.4 & $0.24-0.67$ & \\
USG mass & & & 0.003 \\
\hline Absent & Ref. & \\
Present & 2.75 & $1.40-5.38$ & \\
MMG mass & & & 0.007 \\
\hline Absent & Ref. & \\
\hline Present & 2.23 & $1.24-4.01$ & \\
Suspicious invasion in preoperative biopsy & & & 0.003 \\
\hline Absent & Ref. & \\
\hline Present & 3.96 & $1.59-9.84$ & \\
\hline
\end{tabular}

$\mathrm{OR}$, odds ratio; $\mathrm{Cl}$, confidence interval; $\mathrm{BCS}$, breast-conserving surgery; HER2/neu, human epidermal growth factor receptor 2; USG, breast sonography; MMG, mammography.
(Supplementary Fig. 1, only online). The reason for this was that DCIS underestimation was approximately $40 \%$ among the patients assessed with the previous nomogram, which was $22 \%$ in the validation group. ${ }^{10,15}$ This was due to the difference in patient populations between the previous data and the validation data. The previous study was conducted using data from 2000 to 2008, and the independent cohort used data from patients who were diagnosed from 2009 to 2012. The number of actual DCIS patients increased recently because the proportion of patients who were diagnosed via breast screening increased, which led to an increased number of patients diagnosed with DCIS. In addition,

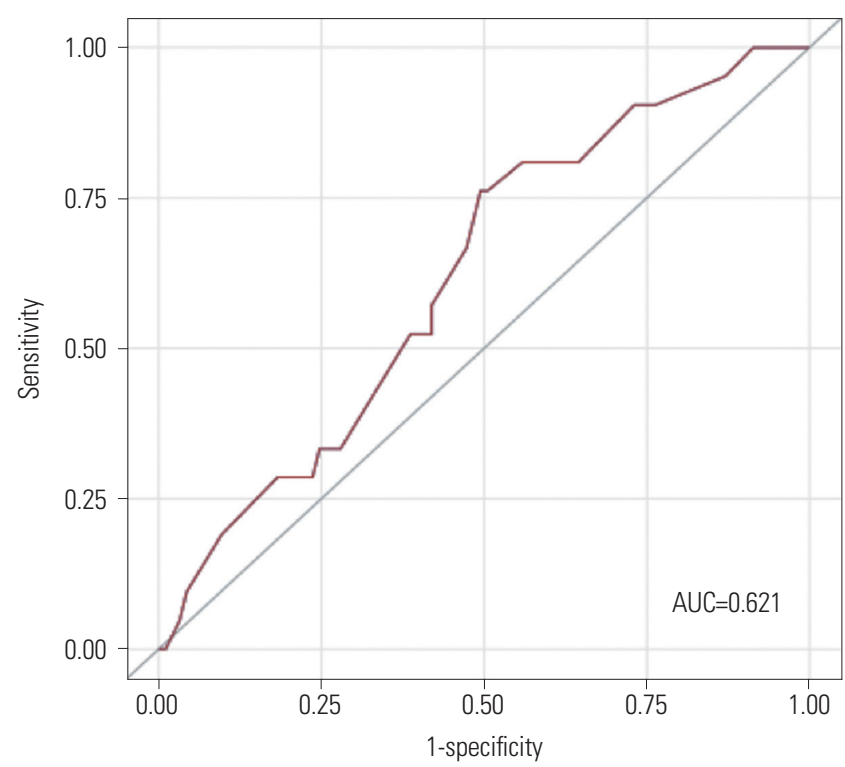

Fig. 3. Receiver operating characteristic curve for external validation with the independent data set $(n=115)$. AUC, area under the curve.

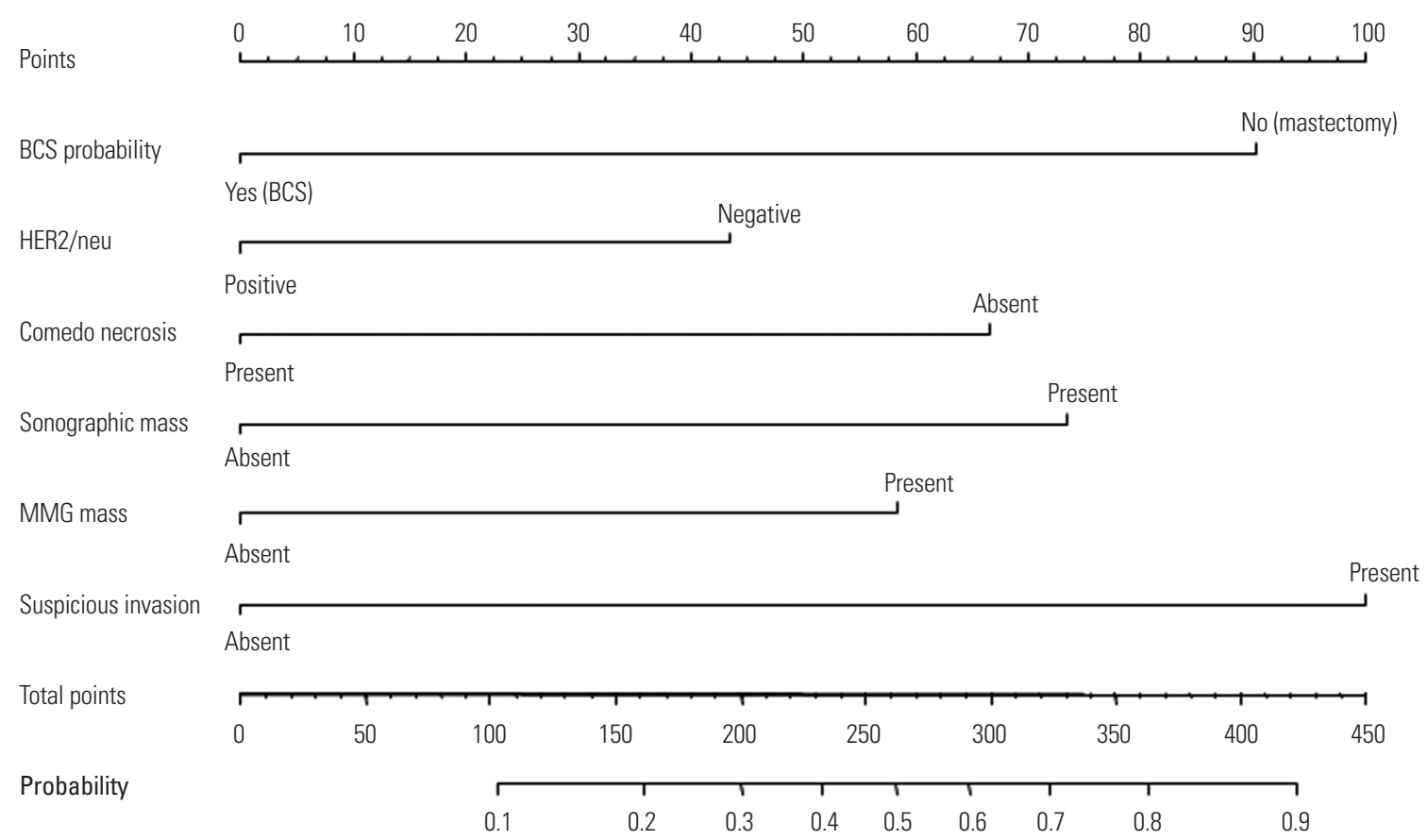

Fig. 2. An updated nomogram. BCS, breast-conserving surgery; HER2/neu, human epidermal growth factor receptor 2; MMG, mammographic. 
diagnostic skills for pathologists may have improved over time, which may have influenced the accuracy of preoperative diagnosis. Hence, the previous nomogram may be over-fitted. Accordingly, we re-analyzed the risk factors that underestimated invasive cancer with DCIS in preoperative diagnosis.

Consequently, the significant predictive factors were sonographic mass, mammographic mass, comedo necrosis, overexpression of HER2/neu, suspicious microinvasion in preoperative examination, and operation type. Most of the patients who underwent mastectomy had breast cancer of large size or multicentricity. Previous studies have attempted to include the size of lesions as a predictor of risk in nomograms. ${ }^{11,13,15}$ Lesion size and multi-centricity on imaging were not used in this study because they are often difficult to measure and inaccurate because of the ambiguity of the extent of microcalcification on mammography, non-circumscribed margin, or hypoechoic microcalcified lesions on ultrasound, as well as background enhancement of magnetic resonance imaging. Instead of lesion size and multi-centricity, we inferred the impact of breast cancer size and multi-centricity based on the operation type. In validation using an external independent cohort, the updated nomogram showed moderate-to-strong discrimination of upgrading preoperative DCIS, and its performance was higher than that of the previous nomogram (AUC for external validations, 0.62 vs. 0.4).

Our previous study identified palpability, calcification on sonography, sonographic mass, previous biopsy methods, and presence of suspicious microinvasion foci as related factors to predict invasive cancer in postoperative pathology. ${ }^{10}$ The palpability and existence of calcification on sonography were not significant in the current study. This may have been due to DCIS being increasingly detected via screening over time. ${ }^{16,17}$

DCIS underestimation on biopsy has been reported to decrease as the amount of tissue acquired increases, and the DCIS underestimation rate in VAB is lower than that in CNB. ${ }^{18}$ The previous nomogram also included biopsy methods as a predictor of risk. ${ }^{10,15}$ Regarding the preoperative biopsy method, VAB/ stereotactic biopsy showed lower upstaging of invasive cancer than CNB in univariate analysis (VAB vs. CNB: $11.3 \%$ vs. $27.4 \%$, $\mathrm{OR}=0.34,95 \% \mathrm{CI}=0.19-0.61, p<0.001)$. However, no significance was found in the multivariate analysis in this study. Hence, preoperative biopsy method was excluded from the predictors of risk in the establishment of the nomogram. Nevertheless, it tended to lower the underestimation of invasive cancer $(\mathrm{OR}=0.56$, 95\% CI=0.29-1.07, $p=0.077$ ). Further study is needed to evaluate the role of VAB in the detection of invasiveness in preoperative DCIS.

Sonographic mass and mammographic mass may be related to each other. Therefore, we checked their relationship using McNemar's test, and they showed different values and low concordance $(p<0.001)$. Hence, both factors were used as predictors of risk.

Some studies have reported that subtypes of DCIS, such as non-cribriform type, papillary type, solid type, and comedo ne- crosis, are related to upstaging to invasive cancer. ${ }^{11}$ In this study, we reviewed the presence of comedo necrosis but not other subtypes, and comedo necrosis showed significant association with pure DCIS after surgery (OR=0.4, 95\% CI=0.24-0.67, $p=0.003$ ).

This study has some limitations. This was a retrospective and single-institution study. The study was analyzed and validated using similar imaging and pathology interpreted in the same institution. This mostly uniform design in a single institution could be a definite advantage. However, pathological and image interpretation methods vary according to institution, and these may not be reflected in our nomogram.

In conclusion, the previous version of the nomogram did not effectively discriminate upstaging of preoperative DCIS in an independent cohort. Our updated version of the nomogram provides more accurate information for predicting upstaging of preoperative DCIS.

\section{ACKNOWLEDGEMENTS}

This research was supported by Basic Science Research Program through the National Research Foundation of Korea (NRF) funded by the Ministry of Education (2016R1D1A1B03934564).

\section{AUTHOR CONTRIBUTIONS}

Conceptualization: Hyung Seok Park. Data curation: Jihong Kim, Sanghwa Kim, and Hyung Seok Park. Formal analysis: Ha Yan Kim, Jihong Kim, Sanghwa Kim, and Hyung Seok Park. Funding acquisition: Hyung Seok Park. Investigation: Jihong Kim, Sanghwa Kim, and Hyung Seok Park. Methodology: Ha Yan Kim, Sanghwa Kim, Jihong Kim, and Hyung Seok Park. Project administration: Hyung Seok Park. Resources: Kwanbum Lee, Jeea Lee, Haemin Lee, Jee Ye Kim, Hyung Seok Park, Seung Il Kim, Young Up Cho, and Byeong-Woo Park. Software: Ha Yan Kim and Hyung Seok Park. Supervision: Hyung Seok Park. Validation: Ha Yan Kim. Visualization: Ha Yan Kim, Sanghwa Kim, Jihong Kim, and Hyung Seok Park. Writing_original draft: Sanghwa Kim and Hyung Seok Park. Writing—review \& editing: Jihong Kim, Sanghwa Kim, and Hyung Seok Park.

\section{ORCID iDs}

Sanghwa Kim Jihong Kim Hyung Seok Park

Ha Yan Kim

Kwanbum Lee

Jeea Lee

Haemin Lee Jee Ye Kim

Seung Il Kim

Young Up Cho

Byeong-Woo Park https://orcid.org/0000-0002-1010-3201 https://orcid.org/0000-0002-6535-4040 https://orcid.org/0000-0001-5322-6036 https://orcid.org/0000-0001-8063-0616 https://orcid.org/0000-0001-6131-3930 https://orcid.org/0000-0003-3145-2205 https://orcid.org/0000-0002-3334-2598 https://orcid.org/0000-0003-3936-4410 https://orcid.org/0000-0001-9673-2748 https://orcid.org/0000-0003-2936-410X https://orcid.org/0000-0003-1353-2607

\section{REFERENCES}

1. Giordano SH, Elias AD, Gradishar WJ. NCCN guidelines updates: breast cancer. J Natl Compr Canc Netw 2018;16(5S):605-10. 
2. Veronesi U, Paganelli G, Viale G, Luini A, Zurrida S, Galimberti V, et al. A randomized comparison of sentinel-node biopsy with routine axillary dissection in breast cancer. N Engl J Med 2003;349: 546-53.

3. Lyman GH, Somerfield MR, Bosserman LD, Perkins CL, Weaver DL, Giuliano AE. Sentinel lymph node biopsy for patients with early-stage breast cancer: American Society of Clinical Oncology Clinical Practice Guideline update. J Clin Oncol 2017;35:561-4.

4. Prendeville S, Ryan C, Feeley L, O'Connell F, Browne TJ, O'Sullivan $\mathrm{MJ}$, et al. Sentinel lymph node biopsy is not warranted following a core needle biopsy diagnosis of ductal carcinoma in situ (DCIS) of the breast. Breast 2015;24:197-200.

5. Sakr R, Bezu C, Raoust I, Antoine M, Ettore F, Darcourt J, et al. The sentinel lymph node procedure for patients with preoperative diagnosis of ductal carcinoma in situ: risk factors for unsuspected invasive disease and for metastatic sentinel lymph nodes. Int J Clin Pract 2008;62:1730-5.

6. Tan EY, Lo ZW, Ang CH, Teo C, Seah MD, Chen JJ, et al. Sentinel lymph node biopsy should be included with the initial surgery for high-risk ductal carcinoma-in-situ. Int Sch Res Notices 2014;2014: 624185.

7. Brennan ME, Turner RM, Ciatto S, Marinovich ML, French JR, Macaskill P, et al. Ductal carcinoma in situ at core-needle biopsy: meta-analysis of underestimation and predictors of invasive breast cancer. Radiology 2011;260:119-28.

8. Sakr R, Antoine M, Barranger E, Dubernard G, Salem C, Daraï E, et al. Value of sentinel lymph node biopsy in breast ductal carcinoma in situ upstaged to invasive carcinoma. Breast J 2008;14:55-60.

9. Coufal O, Selingerová I, Vrtělová P, Krsička P, Gabrielová L, Fabian $\mathrm{P}$, et al. A simple model to assess the probability of invasion in ductal carcinoma in situ of the breast diagnosed by needle biopsy. Biomed Res Int 2014;2014:480840.

10. Park HS, Kim HY, Park S, Kim EK, Kim SI, Park BW. A nomogram for predicting underestimation of invasiveness in ductal carcinoma in situ diagnosed by preoperative needle biopsy. Breast 2013;
22:869-73.

11. Lee SK, Yang JH, Woo SY, Lee JE, Nam SJ. Nomogram for predicting invasion in patients with a preoperative diagnosis of ductal carcinoma in situ of the breast. Br J Surg 2013;100:1756-63.

12. Kondo T, Hayashi N, Ohde S, Yagata H, Yoshida A, Nakamura S, et al. A nomogram associated with high probability of invasive carcinoma on the surgical specimen in patients with preoperative diagnosis of ductal carcinoma in situ of the breast. J Clin Oncol 2014; 32(no. 15_Suppl):1595.

13. Jakub JW, Murphy BL, Gonzalez AB, Conners AL, Henrichsen TL, Maimone $S$ 4th, et al. A validated nomogram to predict upstaging of ductal carcinoma in situ to invasive disease. Ann Surg Oncol 2017;24:2915-24.

14. Meurs CJC, van Rosmalen J, Menke-Pluijmers MBE, Ter Braak BPM, de Munck L, Siesling S, et al. A prediction model for underestimation of invasive breast cancer after a biopsy diagnosis of ductal carcinoma in situ: based on 2892 biopsies and 589 invasive cancers. Br J Cancer 2018;119:1155-62.

15. Park HS, Park S, Cho J, Park JM, Kim SI, Park BW. Risk predictors of underestimation and the need for sentinel node biopsy in patients diagnosed with ductal carcinoma in situ by preoperative needle biopsy. J Surg Oncol 2013;107:388-92.

16. Kang SY, Kim YS, Kim Z, Kim HY, Lee SK, Jung KW, et al. Basic findings regarding breast cancer in Korea in 2015: data from a breast cancer registry. J Breast Cancer 2018;21:1-10.

17. Duffy SW, Dibden A, Michalopoulos D, Offman J, Parmar D, Jenkins J, et al. Screen detection of ductal carcinoma in situ and subsequent incidence of invasive interval breast cancers: a retrospective population-based study. Lancet Oncol 2016;17:109-14.

18. Suh YJ, Kim MJ, Kim EK, Moon HJ, Kwak JY, Koo HR, et al. Comparison of the underestimation rate in cases with ductal carcinoma in situ at ultrasound-guided core biopsy: 14-gauge automated core-needle biopsy vs 8- or 11-gauge vacuum-assisted biopsy. $\mathrm{Br}$ J Radiol 2012;85:e349-56. 